\title{
Heavy Ion Physics with the ATLAS detector
}

\author{
H. Takai \\ Brookhaven National Laboratory, Upton NY 11973 \\ e-mail: takai@bnl.gov \\ Received:
}

\begin{abstract}
Recent results from RHIC experiments suggest that a hot and dense QCD matter, which maybe the quark gluon plasma (QGP), is formed in Gold+Gold collisions at a center mass energy of $200 \mathrm{GeV}$ per colliding nucleon pair. The LHC is planning to accelerate heavy nuclei such as Lead at energies of $2.75 \mathrm{TeV} /$ nucleon. At these energies it will be possible to produce a even higher temperature QCD matter. In addition, hard scattering cross sections will increase significantly and they could be used as probes of the QCD matter. RHIC experiments suggest that hard scattered quarks inside the QGP radiate gluons and therefore modify the jet properties such as energy angular distribution. The ATLAS detector with its large acceptance is ideally suited to detect and study jets from nucleus-nucleus collisions. Initial simulation studies show most of the ATLAS detector will perform well in a high multiplicity environment, including inner detector tracking. Jet reconstruction is possible with an energy resolution close to the high luminosity proton-proton run. In this paper we present a summary of our initial round of simulations studies of the ATLAS detector in the heavy ion environment.
\end{abstract}

PACS: 25.70.Ef; 21.60.Gx; 27.30.+t

\section{Introduction}

In ultra-relativistic central nucleus-nucleus collisions $(b \sim 0 \mathrm{fm})$, an enormous number of virtual partons, dominated by gluons, are freed from the nuclear wave function. These partons should form a quark-gluon-plasma characterized by quark de-confinement and restoration of approximate chiral symmetry. One major advantage of the higher LHC collision energy compared to RHIC is that the initial parton densities are so high that a description of the gluons as color field solutions to the Yang-Mills equation may be relevant. This picture, referred to as the color glass condensate, has had some initial success in describing RHIC data, but would apply more clearly in the $Q^{2}$ regime at the LHC $[1,2]$.

At the LHC energy regime, hard processes namely jets can serve as ideal probes of the hot QCD matter created after the nuclei collide. Because of the expected longer lifetime and higher temperature of the de-confined state these probes could bring direct information of the plasma property. One of the key observables is the measurement of parton probes of the plasma medium via their induced gluon radiation, often referred to as jet quenching $[3,4,5]$. The parton energy loss is directly related to the initial gluon density of the system, which is expected to be over a factor of ten higher than at RHIC. As these partons travel 
through the plasma their energy loss should result in a softening of the final jet fragmentation into hadrons. Given the large calorimetric coverage and the fine segmentation of the ATLAS detector, these probes are most suitable to study the underlying nuclear physics.

Results from the PHENIX experiment at RHIC[6] have shown hints of energy loss effects. PHENIX measures a suppression of high $p_{T} \pi^{0}$ that is attributed to the quenching phenomena. This result can be explained by models that take into account induced gluon radiation in the QGP, for example Vitev et al[7]. The results also point out the critical need to measure jet fragmentation with a known original total jet energy. It is noteworthy that at LHC a larger suppression is expected due to the predicted higher gluon density and that the suppression remains significant for $p_{T}>50 \mathrm{GeV}[7]$.

There is also an excellent opportunity in ATLAS to measure $\gamma$-jet, jet-jet and Z-jet events where one can more fully characterize the modified fragmentation functions. In particular, the $\gamma($ or Z $)$ in $\gamma($ or Z $)$-jet processes provides a control over the away-side jet energy and direction that will allow the physics of quenching to be studied quantitatively and in great detail. The effects of hard gluon radiation on the $\gamma /$ jet energy imbalance and angular distribution can be studied in great detail using the high-statistics p-p data set. The $\gamma$-jet channel requires the identification of a photon. In proton-proton collisions the rejection of $\gamma / \pi^{0}$ is about a factor of three up to a $p_{T}$ of $50 \mathrm{GeV}$. However, the heavy ion environment presents considerable more challenge. $Z^{0}$ production rates have been estimated by Wang and Huang[8]. For $p_{T}$ larger than $40 \mathrm{GeV}$, we expect of the order of $500 Z^{0} \rightarrow \mu^{+} \mu^{-}$events for one month-run. Therefore multiple runs may be required to extract relevant information on jet fragmentation.

Recent theoretical investigations[9] have indicated that charm and bottom quarks propagating through a dense partonic medium will have supressed gluon radiation. Thus the possibility of measuring b-jets in ATLAS would give an important comparison measurement to the light quark and gluon jets. Such measurements would have important implications on gluon shadowing and saturation models. Suppression of quarkonia states is expected in a de-confined medium due to the screening of the long-range attractive potential. We are beginning studies of the ATLAS capabilities to identify $\Upsilon$ states.

To evaluate the detector capabilities for heavy ion physics, simulation studies are under way. We report on early results from these studies. The simulation addresses ATLAS capabilities as far as tracking, jet reconstruction and $\Upsilon$ mass resolution in the heavy ion environment. The event characterization is also an important ingredient for the heavy ion studies and attention was given to that as well. The results presented here are for full simulation of the detector.

\section{Simulation Tools and Event Generator}

Full simulation studies of the ATLAS response to $\mathrm{Pb}+\mathrm{Pb}$ central collisions $(b=0)$ are being performed. The detector geometry description used is the same as for the proton-proton simulation studies. In the interest of time we reduced the rapidity range of simulated tracks to $-3.2<\eta<3.2$ and raised the simulation threshold in the calorimeter to $1 \mathrm{MeV}$. This leaves out the forward 
calorimeter that will be included in the near future. The tracker threshold used in the simulation is the same used as in the proton-proton case and includes delta-rays.

The present studies relies heavily on two generators: HIJING and PYTHIA. HIJING, is used mostly to generate the background of soft particles, although it includes mini-jets and low $p_{T}$ jets. The default HIJING produces a particle density of $(d N / d y)_{c h} \sim 3,500$ mostly of soft $p_{T}$ particles $(<1 G e V / c)$. PYTHIA is used to generate additional components of events such as jets. When possible we also make use of the ATLAS event library generated during the ATLAS Data Challenge 1. For dedicated studies such as upsilon decays a single particle generator is used.

The information flow used in the simulation is illustrated in Fig. 1. HIJING events for impact parameter in the range of $0-15 \mathrm{fm}$ are generated and stored. A procedure for overlaying the event of interest is available.

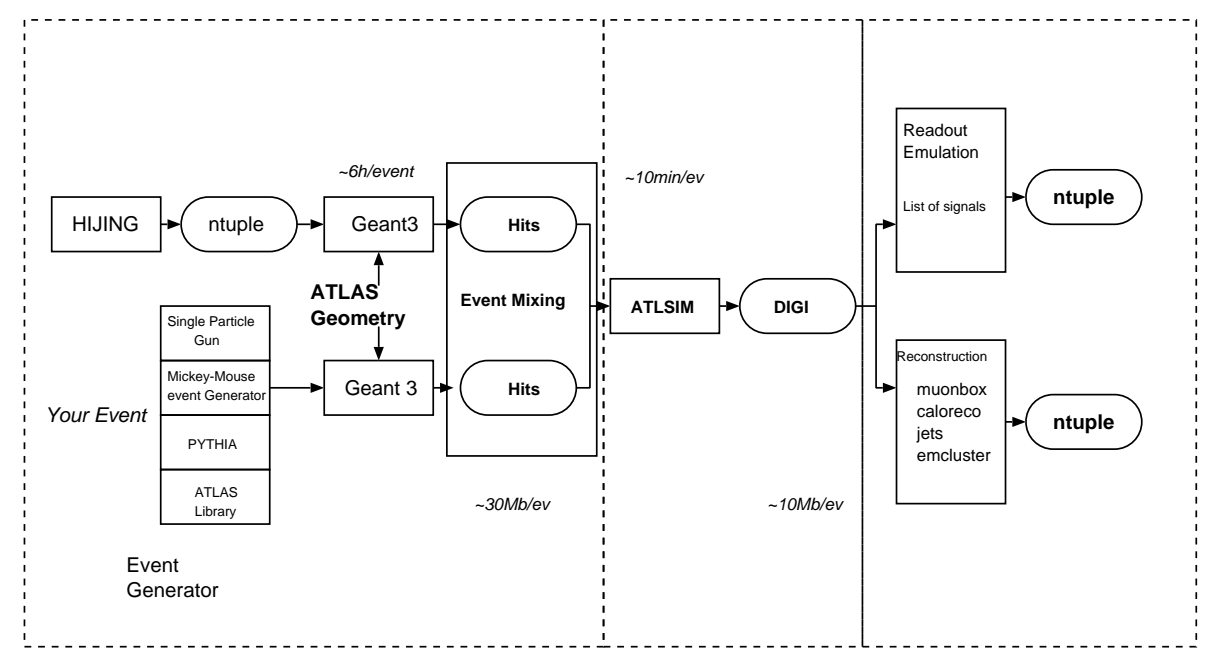

Fig. 1. Information flow in the simulation of heavy ion events.

\section{Detector Occupancies}

Tracking of charged particles in central $\mathrm{Pb}+\mathrm{Pb}$ collisions will be done with the Pixel and SCT detectors. The occupancies for these detectors are shown in Fig 2. As illustrated in the figure the average occupancy for the Pixel system is $\sim 1 \%$. The occupancy for the SCT system is for most part approximately $10 \%$. The error bars in the plots indicate the range of fluctuation in the occupancy, i.e the minimum and the maximum.

In central $\mathrm{Pb}+\mathrm{Pb}$ events most of the calorimeter towers will have hits. Fig. 3 depicts the amount of energy deposited in a typical $\Delta \eta \times \Delta \phi=0.1 \times 0.1$ tower in the Electromagnetic and Hadronic calorimeter systems. The HIJING events deposit most of the energy in the Electromagnetic section of the calorimeter 

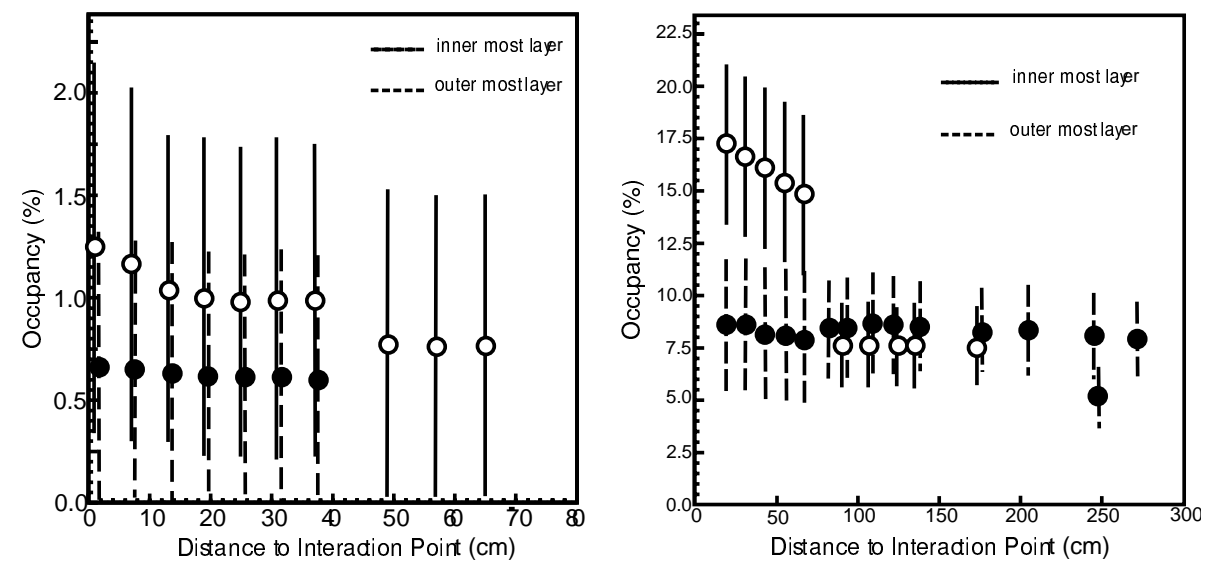

Fig. 2. Occupancy of the (a) Pixel and (b) Silicon strip detector system for central $\mathrm{Pb}-\mathrm{Pb}$ collisions at 5.5. $\mathrm{TeV} /$ nucleon.

leaving the hadronic calorimeter with an average energy deposition of $0.5 \mathrm{GeV}$ per cell. The distribution of energy within the EM calorimeter indicates that the energy is being deposited in the first layers $\left(3 X_{0}\right)$ of the calorimeter.

In high luminosity proton-proton runs the occupancy of the muon spectrometer is dominated by slow neutrons from previous bunch crossings. The long drift time gives detectors in the muon spectrometer "memory" of past events. In heavy ion collisions due to much lower luminosity, undesired hits are from the same event, i.e. $\pi$ and $K$ decays. Full simulations show that the occupancy in the detectors is less than in high luminosity proton runs. Most particles produced in $\mathrm{Pb}+\mathrm{Pb}$ collisions are soft in nature and will be absorbed by the calorimeter. This will allow us to identify and track muons in the spectrometer in the heavy ion environment.

\section{Global Variables and Impact Parameter}

Charged particle multiplicity $\left(N_{c h}\right)$ and charged particle density $\left(d N_{c h} / d \eta\right)$ are the most fundamental observables which will be obtained at the very beginning of the LHC Pb-Pb running. They provide the global properties of the system created in heavy ion collisions and reflect the time integral over a variety of physics processes contributing to the multi-particle production. From these basic measurements one can infer information on the initial conditions like the energy density and the parton density saturation. They are also sensitive to the details of the nuclear structure functions and the interplay of hard and soft processes as well as the effects of parton shadowing and jet quenching. Finally the rescattering processes taking place during later stages of the system evolution and the entropy production throughout the whole evolution of the system also influence the particle production. The other 'day-one' measurements of the total transverse energy $\left(E_{T}\right)$ and the transverse energy per $\eta$ unit $\left(d E_{T} / d \eta\right)$ can ad- 

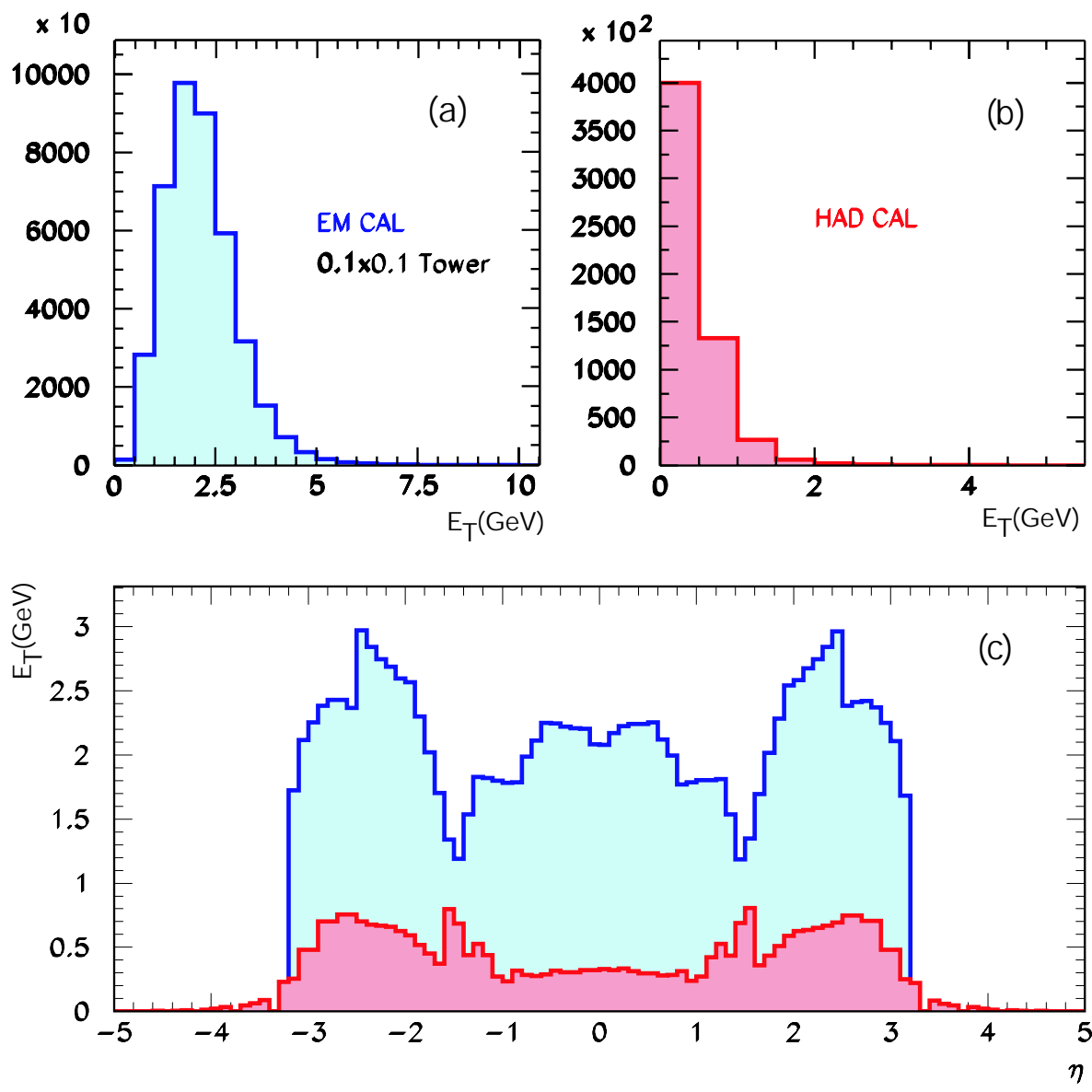

Fig. 3. Distribution of energy deposited in a $\Delta \eta \times \Delta \phi=0.1 \times 01$ tower, for (a) electromagnetic calorimeter and (b) for the hadronic calorimeter. The average energy per tower as function of $\eta$ is shown in (c).

ditionally determine how much of the initially available energy is converted into the transverse degrees of freedom. These measurements provide also means to study the detector performance and estimate the accuracy of the measurements of other observables.

The reconstructed multiplicity distribution $d N_{e v} / d N_{c h}^{r e c}$ is compared to the input HIJING $d N_{e v} / d N_{c h}^{t r u e}$ distribution in Figure 4 . The agreement is very good. The relative reconstruction errors, defined as $\left(N_{c h}^{r e c}-N_{c h}^{\text {true }}\right) / N_{c h}^{\text {true }}$ is about $2 \%$ for central $\mathrm{Pb}-\mathrm{Pb}$ collisions. For most peripheral interactions the accuracy rapidly deteriorates to a value of about $10 \%$ in the relative error. The transverse energy can also be used to measure centrality. Simulation shows tight correlation between $E_{T}$ and $N_{c h}$.

The collision impact parameter is not directly accessible in the experiment. 


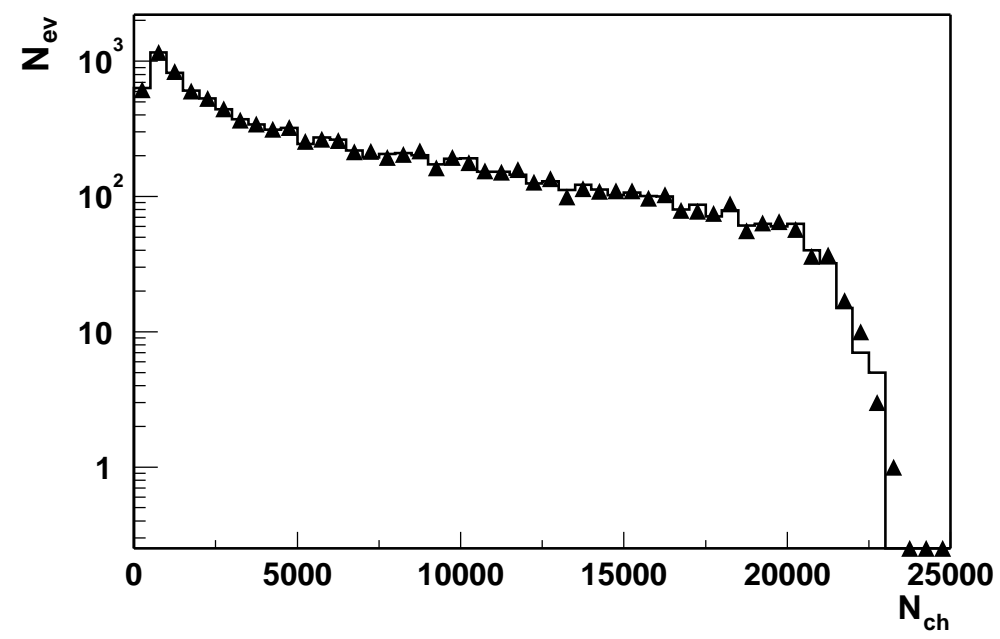

Fig. 4. Comparison of the reconstructed (triangles) and true (histogram) charged particle multiplicity distributions.

Therefore a measurable quantity which correlates to it needs to be identified. The number of pixel or SCT hits monotonically decreases with increasing impact parameter. We can use this correlation to select events with different collision centrality by making cuts on the value of $N_{\text {sig }}$. The monotonic behavior of the correlation assures that samples defined by different ranges in $N_{\text {sig }}$ have well defined, narrow distributions of impact parameters. In Fig. 5 we show the impact parameter resolution obtained on the basis of three distinct measurements: $N_{\text {sig }}$, $E_{T}^{E M}$, and $E_{T}^{H A D}$.

\section{Tracking with the Inner Detector}

The occupancies of the inner detector for $\mathrm{Pb}+\mathrm{Pb}$ central collision allow for track reconstruction of charged particles. The reconstruction can be accomplished using the ATLAS tracking algorithm xKalman++. A single vertex for the tracks can further be assumed because of the low luminosity runs. Work is now under way to optimize xKalman ++ for the heavy ion environment.

The tracking efficiency has been evaluated for central collisions ( $b=0 \mathrm{fm})$. Efficiency figures are shown in Fig 6 as function of the track $p_{T}$ together with the percentage of fake tracks. Tracks with $p_{T}<1 \mathrm{GeV} / \mathrm{c}$ were not reconstructed in the interest of time. The reconstruction efficiency is near $75 \%$ and the fake rate is less than $5 \%$ at $p_{T}<10 \mathrm{GeV}$ but raising for higher $p_{T}$ tracks. One should note that in the simulation we have included effects of electronic readout saturation. Fake tracks originate from random combinatorics of hits and can be eliminated by matching to the energy deposited in the calorimeter for tracks with $p_{T}>10 \mathrm{GeV}$. The exact figures for the rejection are now under study. 


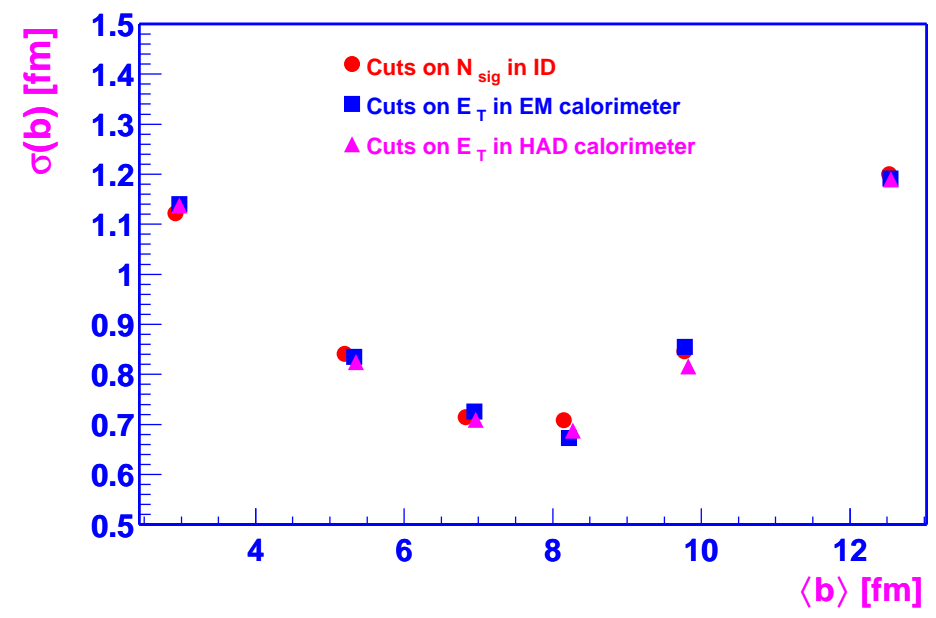

Fig. 5. Impact parameter resolution for three different methods.

The track $p_{T}$ is reconstructed with a precision of $3 \%$ in average in the acceptance of $-2.5<\eta<2.5$. Early studies done for the proton-proton environment indicate that a resolution of $2.5 \%$ is typical for the barrel and worsens for the endcap region. Therefore only a small degradation in the momentum resolution is observed in the heavy ion environment.

\section{Jet Reconstruction}

To study the ability to reconstruct jets in the heavy ion environment PYTHIA jets were overlayed on HIJING events. The jet reconstruction algorithm used in this study is the sliding window, the simplest of the algorithms. Prior to jet reconstruction the average background energy is subtracted from each tower. This procedure however, does not eliminate fluctuations. In the ATLAS calorimeter system the first longitudinal layers could be removed for jet finding and could be important for low energy jets. However, the present study includes all layers for jet finding.

To estimate the jet reconstruction efficiency we count jets found in coincidence with simulated PYTHIA events within a cone of $\Delta R=0.2$. Fake jets in our definition are the ones not having a corresponding PYTHIA jet. However, some fraction of jets comes from HIJING events and therefore the number of fake jets is overestimated. The efficiency plot is shown in the top of Fig. 7. The efficiency reaches $100 \%$ for jet $E_{T}$ larger than $75 \mathrm{GeV}$. Below that the efficiency drops to approximately $60 \%$ for $E_{T}=40 \mathrm{GeV}$.

The jet energy scale is calibrated using ATLAS jet energy calibration procedures described in the Performance Technical Design Report[10]. After the jet energy calibration is applied one can check the result by comparing it to the jet 


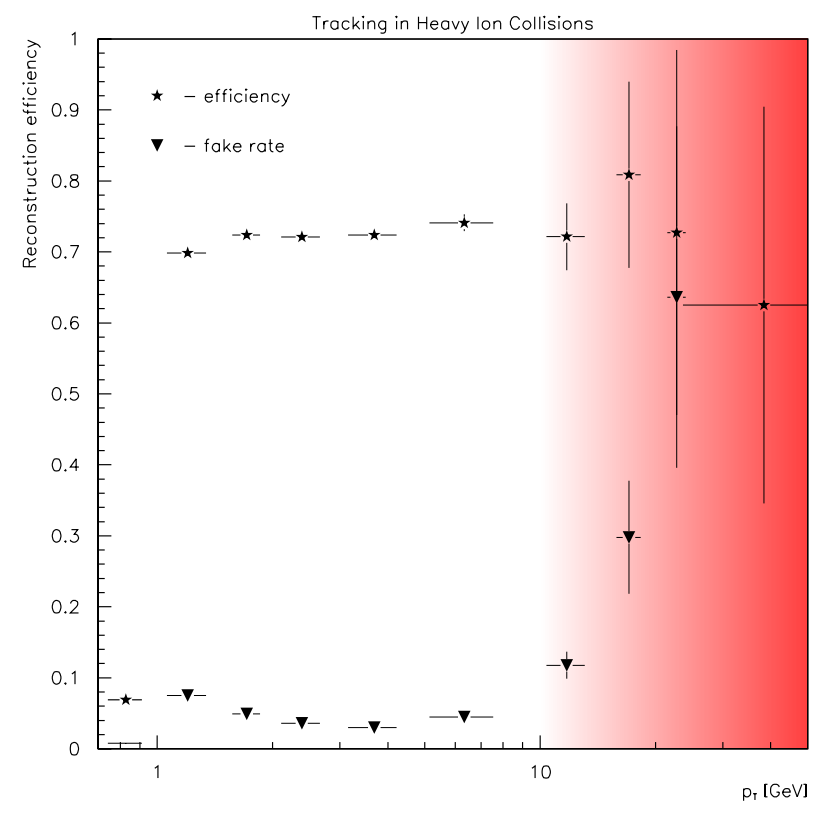

Fig. 6. Track reconstruction efficiency (stars) and fakes (inverted triangles) as function of the track $p_{T}$.

energy at particle level from PYTHIA (with neutrinos subtracted). Such comparison is shown in the bottom of the Fig. 7 for both the jet alone and the jet overlayed on the HIJING background. The worsening introduced by the background has nearly the same effect when pile-up and electronic noise is added to high luminosity proton-proton runs[10]. A good energy resolution will be important for measuring the inclusive cross section and jet energy angular distribution studies $d E_{T}^{j e t}(r) / d R$, where $\mathrm{R}$ is the jet cone radius. Algorithms to extend the jet reconstruction for lower energies is under way.

The study of the differences between heavy quark and light quark quenching can be accomplished if b-jet quarks can be identified. b-jets can be identified by a muon that is collinear with the jet axis and/or by its displaced vertex. We have performed preliminary studies of the possibility of utilizing the displaced vertex algorithm. For this study we used events from the study of associated Higgs production $p p \rightarrow W H$, with $M_{H}=400 \mathrm{GeV}$. Rejection factors $R_{u}$ against u-quark jets are shown as function of the tagging efficiency $\epsilon_{b}$ in Fig. 8 for the proton-proton and central $\mathrm{Pb}-\mathrm{Pb}$ runs. This work was done with standard ATLAS tools used for the study of b-physics potential for ATLAS and has not been optimized for the heavy ion environment. b-tagging efficiency for a muon tag has not been studied yet but it is expected to improve on the rejection of light quark jets as has been demonstrated in similar proton-proton studies.

In the near future the tracking and calorimeter analysis will be merged. This may also reduce the threshold for jet reconstruction. 


\section{Jet fragmentation function}

The measurement of the jet fragmentation function is the most sensitive way to access the magnitude of the quenching effect. In the heavy ion environment track reconstruction efficiency is low because of the large background of soft $p_{T}$ particles. Therefore the determination of the jet fragmentation function via particle momentum measurement is subject to inaccuracies. In ATLAS the reconstruction efficiency of tracks in the jet is $70 \sim 80 \%$ for central impact parameter events. While statistically this might be acceptable the occasional non reconstruction of high $p_{T}$ tracks might lead to biased results. Therefore a background robust technique needs to be found.
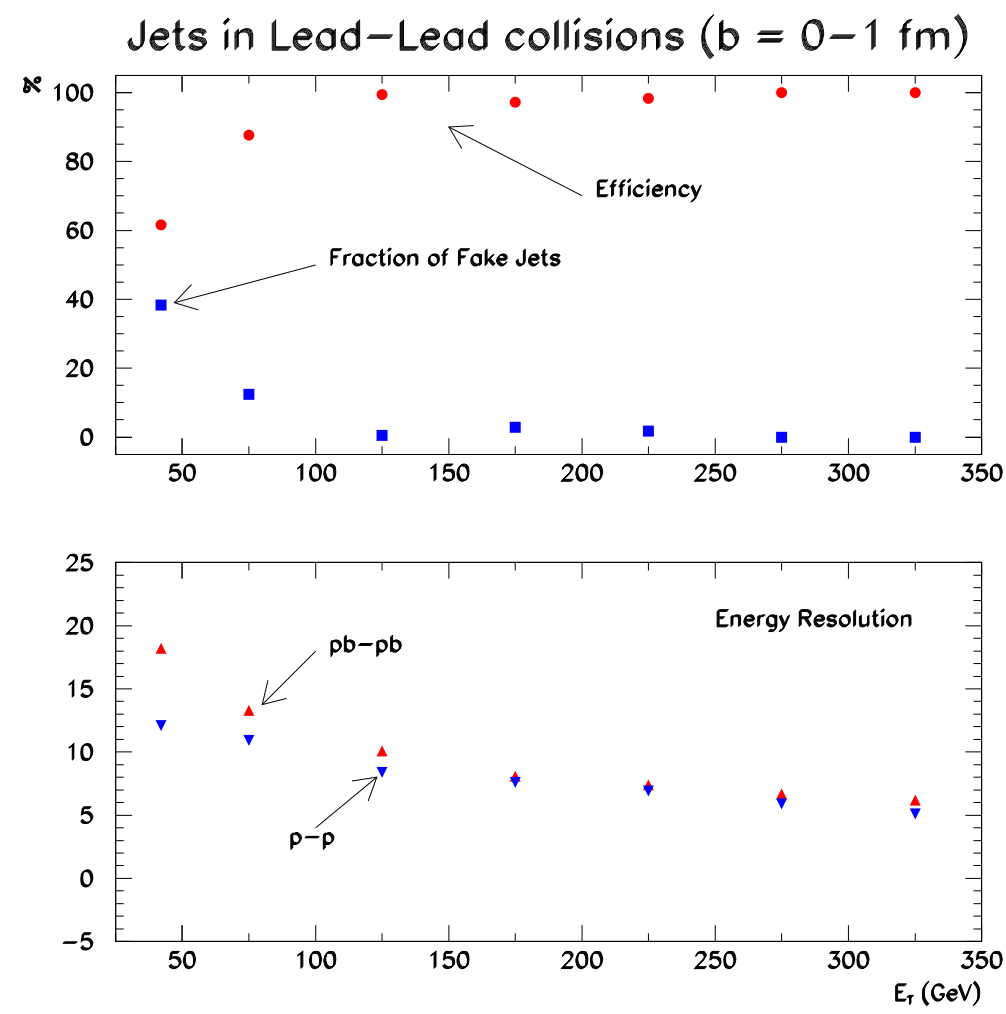

Fig. 7. Top: jet reconstruction efficiency for PYTHIA jets overlayed on HIJING events. Bottom: jet energy resolution for the $\mathrm{Pb}+\mathrm{Pb}$ and proton-proton collisions.

Calorimeters are not subject to reconstruction efficiencies. One could imagine measuring the fragmentation function via isolated and identified electromagnetic clusters, which are in their majority $\pi^{0} \mathrm{~s}$. Simulation studies show, however, that at LHC energies isolated clusters can be found in a small fraction of jets, $\sim 1 \%$.

http://link.springer.de/link/service/journals/10105/index.html 
The majority of jets have EM-clusters overlapping with charged particle tracks. Therefore this technique can only be made in a small sample of jets. This might not be a problem since the expected number of jets is very large.

A compromise technique, and perhaps somewhat non conventional, is to measure the jet core $E_{T}$. The jet core $E_{T}$ is the energy within a cone defined by a $\Delta \eta \times \Delta \phi=0.075 \times 0.075$ in the EM calorimeter and $\Delta \eta \times \Delta \phi=0.1 \times 0.1$ in the Hadronic calorimeter. In Fig. 9 we show the jet core $E_{T}$ energy plotted for different jet energies. In the quenching scenario this quantity should be very sensitive to radiation of energy outside of the jet cone. Recent calculations show that quenching effects could lower the $p_{T}$ of the leading particle by as much as $20-30 \%[11]$. Our studies suggest that sensitivities of less than $10 \%$ can be achieved via the core $E_{T}$ measurement. However the sensitivity will be quantified in future studies.
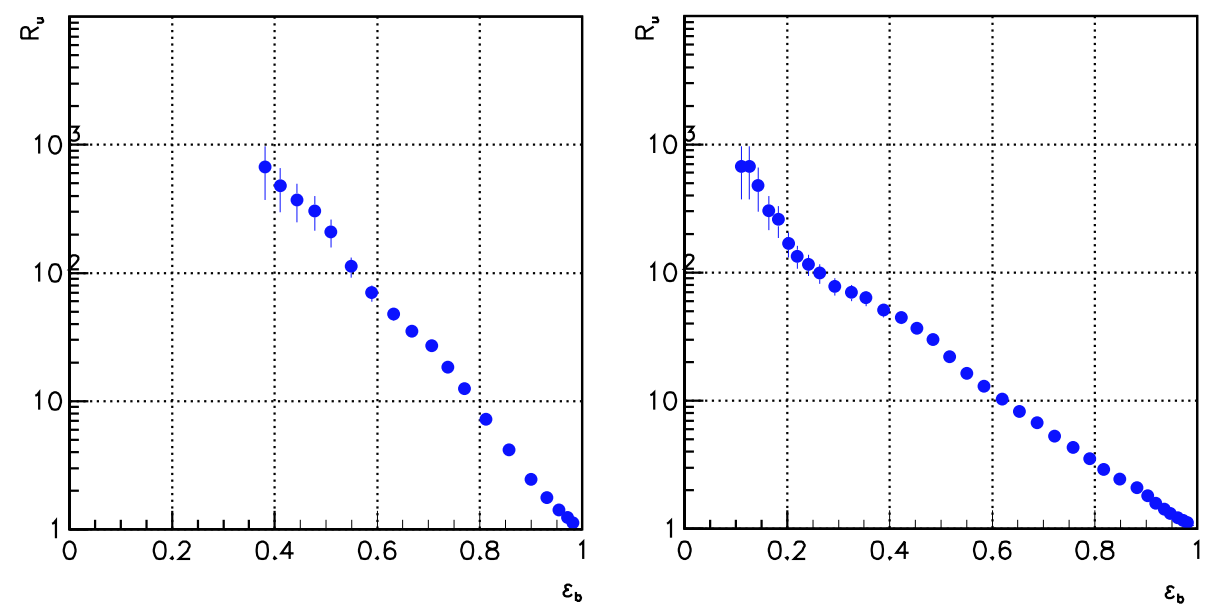

Fig. 8. Rejection factors for u-quarks as function of the b-jet tagging efficiency from displace vertex. Left for proton-proton runs and right for the same events but overlayed to central $\mathrm{Pb}+\mathrm{Pb}$ collisions. In both cases the b-jets are from $\mathrm{WH}$ decays. Jets span a $p_{T}$ range from $\sim 50$ to $\sim 300 \mathrm{GeV} / \mathrm{c}$.

\section{Upsilon reconstruction}

Upsilon mass reconstruction uses both the inner detector and the muon spectrometer. While the muons are identified by the muon spectrometer the mass resolution for this $p_{T}$ range is given by the inner detector. Thus most of the effort in the simulation has been devoted to track matching between both systems. Initial studies are performed with single $\Upsilon$ decays alone. The overlay to heavy ion background is now under way.

The di-muon mass resolution at the $p_{T}$ of interest is completely determined by the inner detector resolution. To study the di-muon mass resolution, $\Upsilon$ 's were generated as single decaying particles in ATLAS and tracked using full 


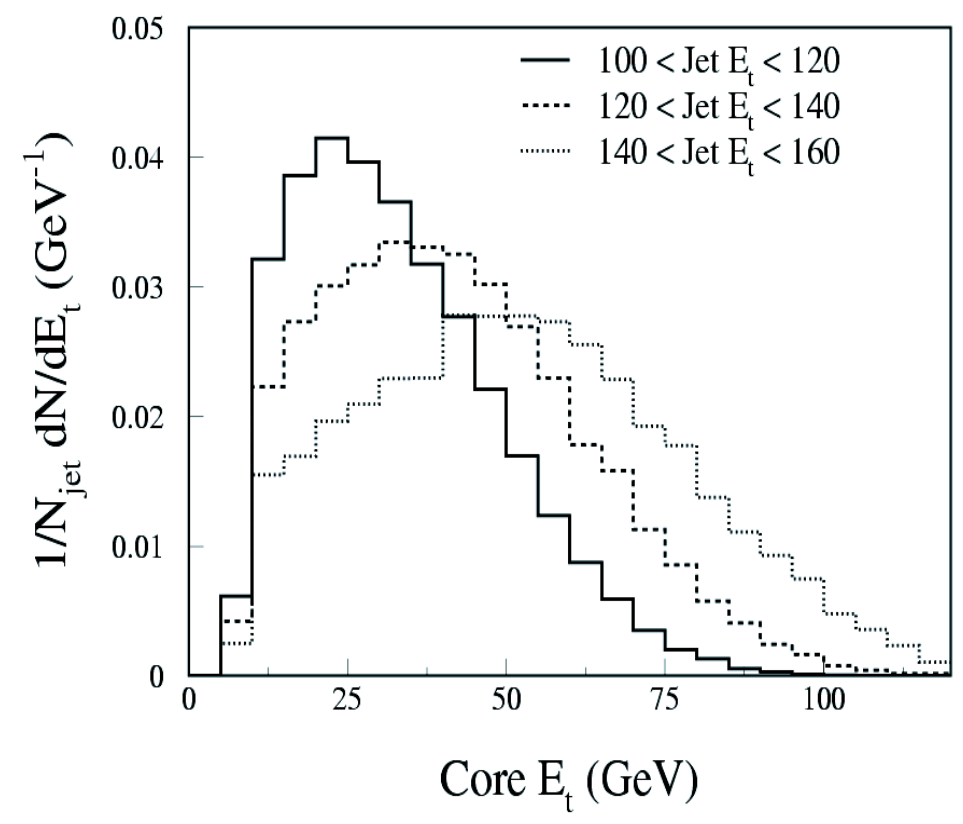

Fig. 9. $E_{T}^{\text {core }}$ distributions obtained from simulations of PYTHIA proton-proton jets of varying energy.

simulation of the detector. The minimum muon $p_{T}$ in ATLAS is $3 \mathrm{GeV}$, and it is dictated by the thickness of the calorimeter system.

The resolution for single $\Upsilon_{\mathrm{S}}$ is $130 \mathrm{MeV}$ in the rapidity range of $-1.4<$ $\eta<1.4$ and worsens to $150 \mathrm{MeV}$ above that rapidity range. The resolution in the ATLAS detector is determined by the multiple scattering in the inner detector system. Simulation done with the mass of the ID set to zero show that resolutions of $20 \mathrm{MeV}$ is seen. However, ATLAS simulations includes a fairly detailed description of the detector hardware. Studies with the inclusion of the heavy ion background are now under way.

\section{Summary}

The ATLAS experiment has been optimized for high $p_{T}$ physics at the LHC proton-proton design luminosity. In central $\mathrm{Pb}-\mathrm{Pb}$ reactions, while the inner silicon layers (Pixel and Silicon Strips) will provide tracking information, the transition radiation tracker (TRT) will have very low efficiency due to high occupancy. The calorimeter has excellent granularity and hadronic energy resolution with good timing resolution and will be fully functional. This will provide the optimal opportunity for measuring jets. The muon spectrometer can be used for efficient muon identification and b-jet tagging using soft decay muons. The proposed heavy ion physics program with the ATLAS detector is outlined as follows: 


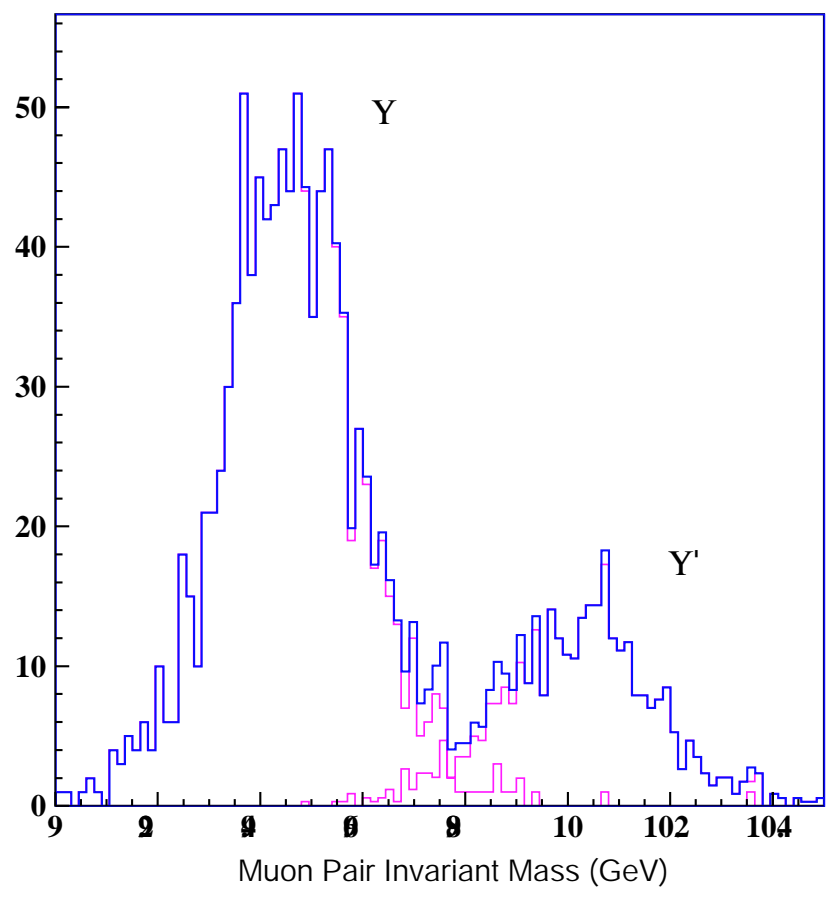

Fig. 10. Reconstructed $\Upsilon$ and $\Upsilon^{\prime}$ states using the muon spectrometer and the

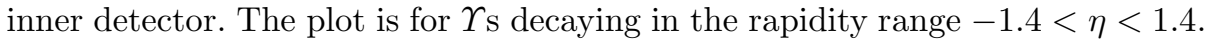
The resolution is $130 \mathrm{MeV}$. In the endcap region $\eta>1.4$ the mass resolution worsens to $145 \mathrm{MeV}$.

- Global variable measurements Measurements of total transverse energy flow $E_{T}$ and $d E_{T} / d \eta$, event multiplicity $N$ and $d N / d \eta$, and elliptic flow will provide a direct measurement of the energy density, reaction dynamics, and will permit the selection of the collision impact parameter. These measurements can be done extremely well with the ATLAS detector.

- Jet quenching studies Hot QCD matter may modify jet properties (e.g. cone radius and energy) because partons may radiate soft gluons in the presence of a dense quark gluon plasma before hadronization. Early RHIC results suggest that quenching may be the cause for the suppression of hadrons at large $p_{T}$. At LHC jet properties can be better measured when compared to RHIC due to the higher collision energy. We are exploring the feasibility of identifying $\gamma-j e t$, jet - jet, and $Z-j e t$ channels in the presence of the heavy ion soft background.

- Heavy quarks Theoretical work indicates that heavy quarks propagating through the QCD medium lose much less energy via gluon bremsstrahlung than light quarks, and this results in less quenching. b-jets in ATLAS, for example, could be tagged by the associated muon or displaced vertex. 
- Quarkonia Screening of the long- range attractive potential is expected within the hot QCD medium. The observation of $\Upsilon$ states suppression is a direct consequence of hot plasma formation.

- Proton-nucleus collisions Proton-nucleus physics at the LHC will focus on the perturbative production of gluons and the modifications of the gluon distribution in the nucleus at low $\mathrm{x}$. We believe that there is an excellent opportunity to study proton-nucleus physics with ATLAS. It will also provide a baseline for the understanding of nucleus-nucleus physics.

- Nucleus-nucleus ultra peripheral collisions The highly Lorentz contracted electric field of heavy nuclei is a source of high energy photonphoton and photon nucleon collisions. Heavy quark photo-production may allow for the determination of the saturation scale within the context of color glass condensate model.

\section{Acknowledgments}

This work has been performed within the ATLAS Collaboration, and we thank collaboration members for helpful discussions. We have made use of the physics analysis framework and tools which are the result of collaboration-wide efforts. This work was supported in part under DOE Contract number DE-AC0298CH10886 .

\section{References}

1. How protons shatter colored glass, A. Dumitru and L. D. McLerran, Nucl. Phys. A700:492-508, 2002.

2. The Color Glass Condensate and Small-x Physics: Four Lectures. L. D. McLerran, hep-ph/0104285, Apr 2001.

3. Jet Quenching In Dense Matter, M. Gyulassy and M. Plumer, Phys. Lett. B243:432-438, 1990.

4. Gluon shadowing and jet quenching in $A+A$ collisions at $\sqrt{s}=200 G e V$, X.N. Wang, M. Gyulassy, Phys. Rev. Lett. 68:1480-1483,1992.

5. Radiative Energy Loss and $p_{T}$ Broadening of High-Energy Partons in Nuclei, R. Baier et al, Nucl. Phys. B484:265-282, 1997.

6. Suppression of Hadrons with Large Transverse Momentum in Central Au $+A u$ Collisions at $\sqrt{s_{n n}}=130$ Gev, K. Adcox et al. Phys. Rev. Lett. 88:022301, 2002.

7. Non-Abelian Energy Loss at Finite Opacity, M. Gyulassy, P. Levai, and I. Vitev, Phys. Rev. Lett. 85:5535-5538, 2000.

8. Study Medium Induced Parton Energy Loss in Gamma + Jet Events of HighEnergy Heavy Ion Collisions, X.N. Wang and Z. Huang, Phys. Rev. C55:3047-3061, 1997.

9. Heavy Quark Colorimetry of QCD Matter, Y.L. Dokshitzer, D.E. Kharzeev, Phys. Lett. B519:199-206, 2001.

10. ATLAS detector and physics performance, Technical Design Report Vol I and II. CERN/LHCC 99-14.

11. Kovner, Alexander and Wiedemann, Urs Achim, hep-ph/0304151 (2003)

http://link.springer.de/link/service/journals/10105/index.html 